\title{
Comprehensive Analysis of the Failure of Intuition in Elementary Rigid Body Dynamics
}

\author{
Bernard Ricardo ${ }^{1,2}$ \\ ${ }^{1}$ Department of Physics and Engineering, NUS High School of Mathematics and Science, Singapore \\ ${ }^{2}$ Natural Sciences and Science Education, National Institute of Education, Singapore \\ Correspondence: Bernard Ricardo, Department of Physics and Engineering, NUS High School of Mathematics \\ and Science, 20 Clementi Ave 1, Singapore. Tel: 65-651-67295. E-mail: nhsbrw@nus.edu.sg
}

Received: December 31, 2015 Accepted: January 8, 2016 Online Published: January 29, 2016

doi:10.5539/apr.v8n1p125

URL: http://dx.doi.org/10.5539/apr.v8n1p125

\begin{abstract}
This article provides a thorough and comprehensive analysis of an elementary problem in rigid body dynamics, involving a projectile and a rod on a smooth table. The problem presented is an extension of a problem taken from an undergraduate physics textbook. This paper presents some generalisations to the problem, namely the unevenness (non-uniformity) of the rod's mass distribution, the elasticity of the collision, and the case where the rod is pivoted. This paper presents nice analytical steps that address some misconceptions in students' way of thinking as well as the failure of intuition when it comes to solving physics problems which necessarily require mathematical approach. The result is quite surprising and counterintuitive as it goes against our intuition with daily experience involving doors and levers.
\end{abstract}

Keywords: failure, intuition, rigid body, dynamics, collision

\section{Introduction}

Dynamics is a branch of classical mechanics concerned with the study of forces and torques and their effect on motion, as opposed to kinematics, which studies the motion of objects without reference to its causes. Isaac Newton defined the fundamental physical laws which govern dynamics in physics, especially his second law of motion. Just as the study of Newtonian dynamics begins by defining a force and its relation to the system's momentum, the study of rotational dynamics starts by defining our analogue to a force - a torque - and its relation to the system's angular momentum.

The analysis of physics problems has often been downgraded into intuitive approach, which although it may work for elementary physics concepts, but is often defied by the actual outcome in a more complex situation. For example, in a linear collision, a recent paper demonstrates that even both physics students and teachers with strong conceptual understanding could wrongly suggest that putting more masses in between two bodies could not possibly increase the maximum portion of the kinetic energy transferred (Ricardo \& Lee, 2015).

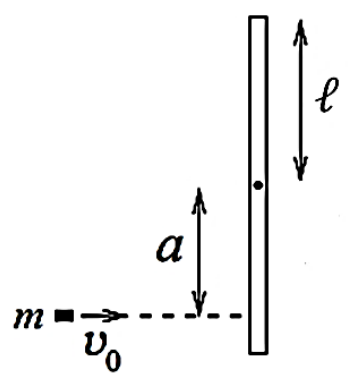

Before

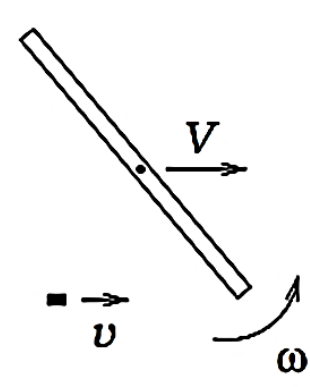

After

Figure 1. A projectile collides perpendicularly with a rigid rod at rest on a smooth horizontal table. 
An elementary problem in rigid body dynamics was discussed in an article written by Lemos (2008) with at least one aspect that does not conform to what intuition suggests. It involves a collision shown in Figure 1, where a projectile collides perpendicularly with a rod on a smooth horizontal table. In this collision, it is natural to intuitively expect that larger $a$ implies larger $\omega$, with $a= \pm \ell$ giving the largest angular speed. This happens as our intuition tells us that the largest angular speed is achieved when the force is applied as far as possible from the rotational axis, or the fulcrum, in this case, the centre of mass of the rod. And this fact is supported by our everyday experience with doors and levers. This particular problem was taken from an undergraduate physics textbook (Resnick et al., 2001, pp.356-357) and the analysis presented in this paper is suitable for undergraduate level.

The analysis for elastic collision has been done in the previous article (Lemos, 2008). This paper presents a more thorough and comprehensive analysis of the case by exploring the unevenness (non-uniformity) of the rod's mass distribution, the elasticity of the collision, and the case where the rod is pivoted. The results are quite surprising and counterintuitive, and are able to provide a deeper understanding for undergraduate physics students in learning rotational dynamics.

\section{Theory}

First, basic concepts needed for the calculations are revisited. It is necessary to understand the appropriate conditions required to use the equations.

\subsection{Conservation of Linear Momentum}

In a closed system (one that does not exchange any matter with its surroundings and is not acted on by external forces $\overrightarrow{\mathbf{F}}_{\text {ext }}$ ) the total linear momentum is constant. This fact, known as the law of conservation of linear momentum, is implied by Newton's laws of motion (Feynman et al., 2013).

$$
\int \overrightarrow{\mathbf{F}}_{\text {ext }} d t=0 \Rightarrow \overrightarrow{\mathbf{p}}_{i}=\overrightarrow{\mathbf{p}}_{f}
$$

where $\int \overrightarrow{\mathbf{F}}_{\text {ext }} d t$ denotes the total external linear impulse, which is necessary to be zero (or negligible) for conservation of linear momentum to be preserved; $\overrightarrow{\mathbf{p}}_{i}$ denotes the initial linear momentum of the system, and $\overrightarrow{\mathbf{p}}_{f}$ denotes the final linear momentum of the system. For a rigid body, like the rod presented in Figure 1, its linear momentum can be obtained by multiplying its mass with the velocity of its centre of mass.

\subsection{Conservation of Angular Momentum}

Similar to linear motion, the law of conservation of angular momentum states that when no (or negligible) external angular impulse by external torque $\overrightarrow{\boldsymbol{\tau}}_{\text {ext }}$ acts on an object or a closed system of objects, the system's angular momentum remains constant (Podolsky, 1966). Commonly the point of collision is taken to be the reference point so as to make the angular impulse acting on each body to be zero. But when a pivot is introduced, the pivot needs to be taken as the reference point so as to make the angular impulse acting on the whole system to be zero.

$$
\int \overrightarrow{\mathbf{\tau}}_{\text {ext }} d t=0 \Rightarrow \overrightarrow{\mathbf{L}}_{i}=\overrightarrow{\mathbf{L}}_{f}
$$

where $\int \overrightarrow{\boldsymbol{\tau}}_{\text {ext }} d t$ denotes the total external angular impulse, which is necessary to be zero (or negligible) for conservation of angular momentum to be preserved; $\overrightarrow{\mathbf{L}}_{i}$ denotes the initial angular momentum of the system, and $\overrightarrow{\mathbf{L}}_{f}$ denotes the final angular momentum of the system.

\subsection{Conservation of Energy}

The law of conservation of energy states that when no (or negligible) work is done by the non-conservative forces $\overrightarrow{\mathbf{F}}_{\mathrm{nc}}$, the total energy in the system is conserved.

$$
\int \overrightarrow{\mathbf{F}}_{\mathrm{nc}} \cdot d \overrightarrow{\mathbf{r}}=0 \Rightarrow E_{i}=E_{f}
$$

where $\int \overrightarrow{\mathbf{F}}_{\text {nc }} \cdot d \overrightarrow{\mathbf{r}}$ denotes the total work done by the non-conservative forces, which is necessary to be zero (or negligible) for conservation of energy to be preserved; $E_{i}$ denotes the initial energy of the system, and $E_{f}$ denotes the final energy of the system. Note that some non-conservative forces may exist in a system, but as long as they do not perform any work on the system, conservation of energy still holds.

\subsection{Moment of Inertia}

The moment of inertia can be defined as the "laziness of an object to change its angular velocity". It is a property of a rigid body, which can be obtained by integration or by making use of the symmetrical property of the shape using Ricardo's scaling method (Ricardo, 2015). The moment of inertia of a uniform rod of mass $m$ and length $2 \ell$ about its centre of mass is given by $I_{0}=\frac{1}{12} m(2 \ell)^{2}=\frac{1}{3} m \ell^{2}$. And if a rotational axis is located at distance $x$ 
away from the centre of mass of the rod, its moment of inertia about the axis can be obtained by using the parallel axis theorem,

$$
I=I_{0}+m x^{2}=\frac{1}{3} m \ell^{2}+m x^{2}
$$

\subsection{Coefficient of Restitution}

Suppose a projectile of mass $m$ and linear velocity $v_{0}$ collides elastically and perpendicularly with a rigid rod of mass $M$ and length $2 \ell$ having initial linear velocity $v_{1}$ in the same direction as $v_{0}$, on a smooth horizontal table. The mass of the rod is assumed to be uniformly distributed.

Let $V$ and $v$ indicate the final linear velocities of the rod's centre of mass and the projectile, respectively, and let $\omega$ indicate the final angular velocity of the rod (Figure 2). For all linear velocities involved, positive sign signifies rightward motion and negative sign leftward motion. For the angular velocity, positive sign signifies anticlockwise rotation and negative sign signifies clockwise rotation.

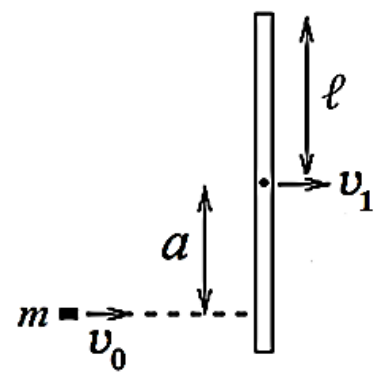

Before

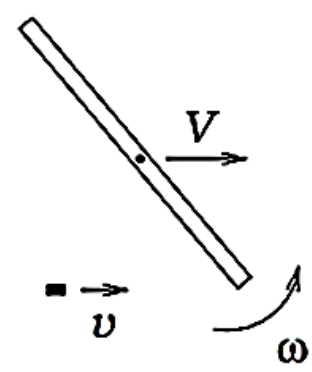

After

Figure 2. Generalisation of the problem: the initial linear velocity of the rod's centre of mass is $v_{1}$

Assuming elastic collision, applying conservation laws in (1), (2), and (3), and by using the moment of inertia expressed in (4) yields

$$
\begin{gathered}
m v_{0}+M v_{1}=m v+M V \\
M v_{1} a=M V a-\left(\frac{1}{3} M \ell^{2}\right) \omega \\
\frac{1}{2} m v_{0}{ }^{2}+\frac{1}{2} M v_{1}{ }^{2}=\frac{1}{2} m v^{2}+\frac{1}{2} M V^{2}+\frac{1}{2}\left(\frac{1}{3} M \ell^{2}\right) \omega^{2}
\end{gathered}
$$

From equations (5) and (7),

$$
\begin{gathered}
m\left(v_{0}-v\right)\left(v_{0}+v\right)=M\left(V-v_{1}\right)\left(V+v_{1}\right)+3 M\left(V-v_{1}\right)^{2} \frac{a^{2}}{\ell^{2}} \\
\left(v_{0}+v\right)=\left(V+v_{1}\right)+3\left(V-v_{1}\right) \frac{a^{2}}{\ell^{2}} \\
v_{0}-v_{1}=V-v+3\left(V-v_{1}\right) \frac{a^{2}}{\ell^{2}}
\end{gathered}
$$

Coefficient of restitution characterises the type of one-dimensional collision (Thornton, 1997). It is commonly defined as $\varepsilon=\frac{(v-v)}{\left(v_{0}-v_{1}\right)}$. This definition does not account for the rotation of the rod, and hence its value is not normalised - it is not equal to one when the collision is elastic, neither is it equal to zero when the collision is inelastic (i.e. the projectile sticks to the rod after the collision instantaneously.). To normalise it, the coefficient of restitution in this specific case can be defined as

$$
\varepsilon=\frac{(V-v)+3\left(V-v_{1}\right) \frac{a^{2}}{\ell^{2}}}{\left(v_{0}-v_{1}\right)}
$$


This is valid to characterise the elasticity of the collision since the value of coefficient of restitution is invariant across the frames of reference. For instance, in a frame moving to the left with speed $u$, all the linear velocities in equation (9) will need to be added by $u$, and hence the value of $\varepsilon$ remains unchanged.

From equation (8), it is obvious that using the definition in (9) for an elastic collision, $\varepsilon=1$. For an inelastic collision, the projectile sticks to the rod, i.e. $v=V+\omega a=V+3\left(V-v_{1}\right) \frac{a^{2}}{\ell^{2}}$, and hence $\varepsilon=0$. Therefore, for any dissipative collision, $\varepsilon \in(0,1)$.

\section{Analysis}

The following three sections present generalisations to the case in Figure 1.

\subsection{First Generalisation: Unevenness (Non-uniformity) of the Rod}

Consider the case where $v_{1}=0$ and the collision is elastic. To generalise the problem, let the centre of mass of the rod be located at distance $x$ away (taken to be a non-negative value) from the centre of the rod, $x \in[0, \ell]$, and its moment of inertia about its centre of mass be $I_{\mathrm{CM}}$ (Figure 3 ). The point of collision is at $a \in[-\ell, \ell]$.

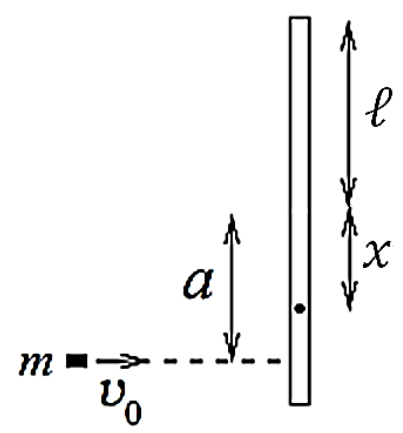

Figure 3. First generalisation: unevenness (non-uniformity) of the rod

As a result of collision, the projectile and the centre of mass of the rod will move horizontally - since there is only horizontal force acting on them during the short time of collision - and at the same time, the rod will rotate about its centre of mass.

Using the sign convention described in section 2.5, applying equations (1), (2), and (3),

$$
\begin{gathered}
m v_{0}=m v+M V \\
M V(a-x)=I_{\mathrm{CM}} \omega \\
\frac{1}{2} m v_{0}^{2}=\frac{1}{2} m v^{2}+\frac{1}{2} M V^{2}+\frac{1}{2} I_{\mathrm{CM}} \omega^{2}
\end{gathered}
$$

yields

$$
\begin{aligned}
& v=\frac{(m-M) I_{\mathrm{CM}}+M m(a-x)^{2}}{(m+M) I_{\mathrm{CM}}+M m(a-x)^{2}} v_{0} \\
& V=\frac{2 m I_{\mathrm{CM}}}{(m+M) I_{\mathrm{CM}}+M m(a-x)^{2}} v_{0} \\
& \omega=\frac{2 M m(a-x)}{(m+M) I_{\mathrm{CM}}+M m(a-x)^{2}} v_{0}
\end{aligned}
$$

It is somewhat clear from equation (11) that there is no way for the rod to move leftward as the expression always yields positive number. However, equation (10) indicates that the projective will move leftward if $m<\frac{M}{1+\frac{M(a-x))^{I}}{I_{\mathrm{CM}}}}$, and it will stop moving after the collision if the equality holds. Moreover from equation (12), the rod will rotate clockwise if $a<x$, and it will stop rotating after the collision if the equality holds. This fact is rather obvious: the rod will rotate clockwise if the projectile hits the rod a point located higher than the centre of mass. 
The question to be addressed in this paper is "Where should the projectile hit for the rod to acquire maximum angular speed?" To answer this, the first and second derivatives of $\omega$ with respect to $a$ should be explored.

$$
\begin{aligned}
\frac{d \omega}{d a} & =\frac{2 M m v_{0}}{\left[(m+M) I_{\mathrm{CM}}+M m(a-x)^{2}\right]^{2}}\left((m+M) I_{\mathrm{CM}}-M m(a-x)^{2}\right) \\
\frac{d^{2} \omega}{d a^{2}} & =-\frac{(2 M m)^{2} v_{0}(a-x)}{\left[(m+M) I_{\mathrm{CM}}+M m(a-x)^{2}\right]^{3}}\left[3(m+M) I_{\mathrm{CM}}-M m(a-x)^{2}\right]
\end{aligned}
$$

The first derivative goes to zero if

$$
a=x \pm \sqrt{\frac{(m+M) I_{\mathrm{CM}}}{M m}}
$$

It is also obvious that $\frac{d^{2} \omega}{d a^{2}}<0$ and $\omega>0$ for $a=x+\sqrt{\frac{(m+M) I_{C M}}{M m}} ; \frac{d^{2} \omega}{d a^{2}}>0$ and $\omega<0$ for $a=x-\sqrt{\frac{(m+M) I_{C M}}{M m}}$, which essentially correspond to the local maxima of the angular speed. The corresponding expressions for $\omega$ are

$$
\omega_{\max }= \pm \sqrt{\frac{M m}{(m+M) I_{\mathrm{CM}}}} v_{0}
$$

Note that since $a \in[-\ell, \ell]$ and $x$ is a non-negative value, for $a=x+\sqrt{\frac{(m+M) t_{\mathrm{c}}}{M m}}$ to exist it is necessary to have

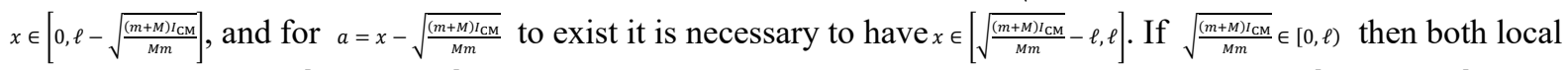
maxima exist for $x \in\left[0, \ell-\sqrt{\frac{(m+M)_{c M}}{M m}}\right]$. If $\sqrt{\frac{(m+M) c_{\mathrm{CM}}}{M m}} \in[\ell, 2 \ell]$ then only one local maximum exist for $x \in\left[\sqrt{\frac{(m+M)_{(\mathrm{CM}}}{M m}}-\ell, \ell\right]$ whose value never falls below the global maximum.

For instance, consider a particular case where the rod is uniform, $x=0, I=\frac{1}{3} m \ell^{2}$ (from equation (4)), equations (13) and (14) yield

$$
\begin{gathered}
a= \pm \ell \sqrt{\frac{m+M}{3 m}} \\
\omega_{\max }= \pm \sqrt{\frac{3 m}{m+M}} \frac{v_{0}}{\ell}
\end{gathered}
$$

with a condition that $\ell \sqrt{\frac{m+M}{3 m}} \leq \ell$ or $M \leq 2 m$. This is counterintuitive to many students, even those with good conceptual understanding, as the maximum angular speed is attained not when the projectile hits the rod's end. In fact, if $a= \pm \ell$, equation (12) gives $\omega= \pm \frac{6 m}{4 m+M} \frac{v_{0}}{\ell}$, which is at most equal to the one obtained in equation (16). To prove that,

$$
\frac{3 m}{m+M}-\left(\frac{6 m}{4 m+M}\right)^{2}=\frac{3 m(2 m-M)^{2}}{(4 m+M)^{2}(m+M)} \geq 0
$$

The equality is achieved when $M=2 m, a= \pm \ell$, i.e. the greatest angular speed is attained when the projectile hits the rod at one end. When $M>2 m$, no local maximum can be found and the rod should be hit at one end, $a= \pm \ell$, to attain the greatest angular speed, $\omega_{\max }= \pm \frac{6 m}{4 m+M} \frac{v_{0}}{\ell}$.

Students also very often argue based on intuition that the maximum angular speed of the rod is attained when the projectile stops moving immediately after the collision, so as to give off its whole kinetic energy to the rod. This misconception is similar to the common one in the case of one-dimensional collision between two blocks (Ricardo \& Lee, 2015). Substituting equation (13) to equations (10) and (11), $v=V=\frac{m}{m+M} v_{0}$, indicates that the maximum angular speed of the rod is attained when the projectile and the centre of mass of the rod move with identical linear velocities after the collision. This is similar to a case of inelastic collision in the absence of rotation. However, the supposedly dissipated energy has now appeared in the form of the rotational kinetic energy. Note that this is true even for the case where the rod is not uniform.

On the other hand, if the rod is hit at its centre of mass, $a=x$, there will be no rotation involved, and the collision is similar to one-dimensional collision between two blocks, $v=\frac{m-M}{m+M} v_{0}, V=\frac{2 m}{m+M} v_{0}$, and $\omega=0$. 


\subsection{Second Generalisation: Elasticity of The Collision}

Consider the case where $v_{1}=0$ and the mass of the rod is uniformly distributed, i.e. $x=0, I_{\mathrm{CM}}=\frac{1}{3} M \ell^{2}$ (Figure 4). The point of collision is at $a \in[-\ell, \ell]$. It shall be assumed that the coefficient of restitution is $\varepsilon$, where $\varepsilon \in[0,1]$, inclusive of the two extreme cases: elastic and inelastic collisions.

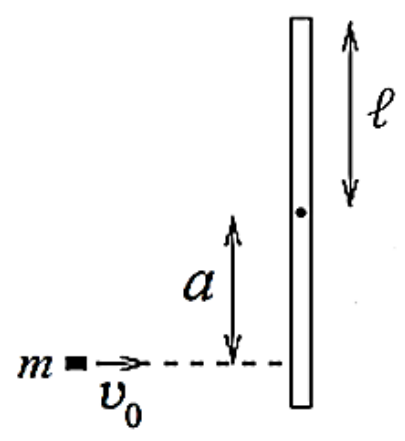

Figure 4. Second generalisation: elasticity of the collision

As a result of collision, the projectile and the centre of mass of the rod will move horizontally - since there is only horizontal force acting on them during the short time of collision - and at the same time, the rod will rotate about its centre of mass.

Using the sign convention described in section 2.5, applying equations (5), (6), and (9), with $v_{1}=0$,

$$
\begin{gathered}
m v_{0}=m v+M V \\
0=M V a-\left(\frac{1}{3} M \ell^{2}\right) \omega \\
\varepsilon=\frac{(V-v)+3 V \frac{a^{2}}{\ell^{2}}}{v_{0}}
\end{gathered}
$$

yields

$$
\begin{gathered}
v=\frac{m\left(1+\frac{3 a^{2}}{\ell^{2}}\right)-M \varepsilon}{m\left(1+\frac{3 a^{2}}{\ell^{2}}\right)+M} v_{0} \\
V=\frac{m(1+\varepsilon)}{m\left(1+\frac{3 a^{2}}{\ell^{2}}\right)+M} v_{0} \\
\omega=\frac{a}{m\left(1+\frac{3 a^{2}}{\ell^{2}}\right)+M} \frac{3 m(1+\varepsilon) v_{0}}{\ell^{2}}
\end{gathered}
$$

It is somewhat clear from equation (18) that there is no way for the rod to move leftward as the expression always yields positive number. However, equation (17) indicates that the projectile will move leftward if $m\left(1+\frac{3 a^{2}}{\ell^{2}}\right)<M \varepsilon$, and it will stop moving after the collision if the equality holds. Moreover, from equation (19), the rod will rotate clockwise if $a<0$, and it will stop rotating if the equality holds, which agrees to common sense.

To find the local maxima of the angular speed, the first and second derivatives of $\omega$ with respect to $a$ should be explored.

$$
\frac{d \omega}{d a}=\frac{3 m(1+\varepsilon) v_{0}}{\ell^{2}} \frac{M+m-\frac{3 m a^{2}}{\ell^{2}}}{\left[m\left(1+\frac{3 a^{2}}{\ell^{2}}\right)+M\right]^{2}}
$$




$$
\frac{d^{2} \omega}{d a^{2}}=-\frac{54 m^{2} a(1+\varepsilon) v_{0}}{\ell^{4}} \frac{M+m-\frac{m a^{2}}{\ell^{2}}}{\left[m\left(1+\frac{3 a^{2}}{\ell^{2}}\right)+M\right]^{3}}
$$

The first derivative goes to zero if

$$
a= \pm \ell \sqrt{\frac{M+m}{3 m}}
$$

and $\frac{d^{2} \omega}{d a^{2}}$ is clearly negative when $a$ takes positive root and positive when $a$ takes negative root, which essentially correspond to the local maxima of the angular speed. The result is surprising, and again, counterintuitive as regardless of the elasticity of the collision, the projectile has to hit the rod at a point $a= \pm \ell \sqrt{\frac{M+m}{3 m}}$ from the centre of the rod to maximise its angular speed. In general, these points are not the rod's ends except if $M=2 \mathrm{~m}$. Since this result is not even affected by the coefficient of restitution, it still holds for the extreme two cases: $\varepsilon=1$ (elastic collision) and $\varepsilon=0$ (inelastic collision).

The corresponding expressions for $\omega$ are given by

$$
\omega_{\max }= \pm \sqrt{\frac{3 m}{M+m}} \frac{(1+\varepsilon) v_{0}}{2 \ell}
$$

which is identical to equation (16) for elastic case. In fact, if $a= \pm \ell$, equation (19) gives $\omega= \pm \frac{6 m}{4 m+M} \frac{(1+\varepsilon) v_{0}}{2 \ell}$, which is at most equal to the expression in equation (21). This fact has been proven in section 3.1, $\frac{3 m}{M+m}-\left(\frac{6 m}{4 m+M}\right)^{2} \geq 0$. The necessary condition that needs to be fulfilled is $|a| \leq \ell$ or $M \leq 2 m$. When $M=2 m$, the local maxima occur at both ends of the rod and $\omega_{\max }= \pm \frac{(1+\varepsilon) v_{0}}{2 \ell}$. If $M>2 m$, no local maximum could be found and the rod should be hit at one of its ends, regardless of the elasticity of the collision.

As mentioned earlier, students may also argue based on intuition that the maximum angular speed of the rod is attained when the projectile stops moving immediately after the collision, so as to give off all its whole kinetic energy to the rod. This is clearly a misconception, similar to the case in section 3.1. Substituting equation (20) to equations (17) and (18), $v=\frac{M(1-\varepsilon)+2 m}{M+m} \frac{v_{0}}{2}$ and $V=\frac{m(1+\varepsilon)}{M+m} \frac{v_{0}}{2}$. It is interesting to see that for an elastic collision, $v=V=$ $\frac{m}{M+m} v_{0}$, i.e. the projectile and the centre of mass of the rod move with identical linear velocities after the collision, similar to a case of inelastic collision in the absence of rotation. For an inelastic collision, $V$ is half of the elastic case, and $v=V+\frac{v_{0}}{2}=V+\omega_{\max } a$, signifying that the projectile sticks to the rod instantaneously. 3.3 Third Generalisation: The Rod is Pivoted.

Consider the case where $v_{1}=0$, the collision is elastic, and the mass of the rod is uniformly distributed, i.e. $x=0$, $I_{\mathrm{CM}}=\frac{1}{3} M \ell^{2}$. To generalise the problem, let the rod be pivoted at a distance $x$ away (taken to be a non-negative value) from its centre (Figure 5). The point of collision is at $a \in[-\ell, \ell]$.

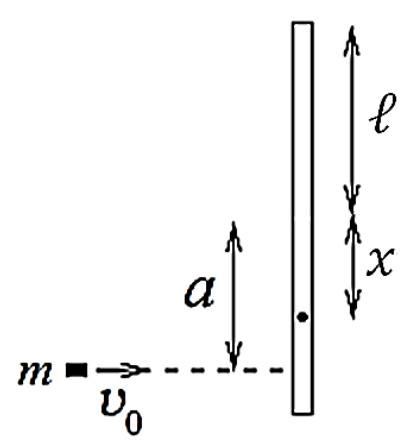

Figure 5. Third generalisation: the rod is pivoted 
As a result of collision, the projectile will move horizontally - since there is only horizontal force acting on them during the short time of collision - and the rod will rotate about the pivot.

In this case, equation (1) is no longer valid as an external force from the pivot is being introduced. Equation (2) can only be applied by taking the pivot as the reference point, for otherwise there will be a non-negligible angular impulse on the system acted by the pivot. Equation (3) still holds as the external force from the pivot performs no (or negligible) work. Using the sign convention in section 2.5., applying equations (2) and (3)

$$
\begin{gathered}
m v_{0}(a-x)=m v(a-x)+\left(\frac{1}{3} M \ell^{2}+M x^{2}\right) \omega \\
\frac{1}{2} m v_{0}^{2}=\frac{1}{2} m v^{2}+\frac{1}{2}\left(\frac{1}{3} M \ell^{2}+M x^{2}\right) \omega^{2}
\end{gathered}
$$

yields

$$
\begin{aligned}
& v=\frac{m(a-x)^{2}-\left(\frac{1}{3} M \ell^{2}+M x^{2}\right)}{m(a-x)^{2}+\left(\frac{1}{3} M \ell^{2}+M x^{2}\right)} v_{0} \\
& \omega=\frac{2 m(a-x)}{m(a-x)^{2}+\left(\frac{1}{3} M \ell^{2}+M x^{2}\right)} v_{0}
\end{aligned}
$$

It is somewhat clear from equation (23) that the rod will not rotate if $a=x$. If $a>x$ then the rod will rotate anticlockwise, and if $a<x$ then the rod will rotate clockwise.

To maximise the angular speed of the rod, the first and second derivatives of $\omega$ with respect to $a$ need to be explored.

$$
\begin{gathered}
\frac{d \omega}{d a}=2 m v_{0} \frac{\left(\frac{1}{3} M \ell^{2}+M x^{2}\right)-m(a-x)^{2}}{\left[m(a-x)^{2}+\left(\frac{1}{3} M \ell^{2}+M x^{2}\right)\right]^{2}} \\
\frac{d^{2} \omega}{d a^{2}}=4 m^{2} v_{0}(a-x) \frac{m(a-x)^{2}-\left(M \ell^{2}+3 M x^{2}\right)}{\left[m(a-x)^{2}+\left(\frac{1}{3} M \ell^{2}+M x^{2}\right)\right]^{3}}
\end{gathered}
$$

The first derivative goes to zero if

$$
a=x \pm \sqrt{\frac{M}{3 m}\left(\ell^{2}+3 x^{2}\right)}
$$

It is also obvious that $\frac{d^{2} \omega}{d a^{2}}<0$ and $\omega>0$ for $a=x+\sqrt{\frac{M}{3 m}\left(\ell^{2}+3 x^{2}\right)}, \frac{d^{2} \omega}{d a^{2}}>0$ and $\omega<0$ for $a=x-\sqrt{\frac{M}{3 m}\left(\ell^{2}+3 x^{2}\right)}$, which essentially correspond to the local maxima of the angular speed. Substituting equation (24) to equation (22), the result also shows that the maximum angular speed will be attained if the projectile stops moving after the collision, which is intuitive since all its kinetic energy is converted into the rod's rotational kinetic energy in this case. Similar to the previous cases, interestingly these points are not the rod's ends.

The corresponding expressions for $\omega$ are given by

$$
\omega_{\max }= \pm \sqrt{\frac{m}{\frac{1}{3} M \ell^{2}+M x^{2}}} v_{0}
$$

Note that since $-\ell \leq a \leq \ell$, for two local maxima to be found, it is necessary to have $-\ell \leq x-\sqrt{\frac{M}{3 m}\left(\ell^{2}+3 x^{2}\right)}$ and $x+\sqrt{\frac{M}{3 m}\left(\ell^{2}+3 x^{2}\right)} \leq \ell$. If only one of them is fulfilled then only one local maximum exists, and if none of them is fulfilled then no local maximum can be found.

The first inequality is equivalent to $f(x)=3(M-m) x^{2}-6 m \ell x+(M-3 m) \ell^{2} \leq 0$. Its discriminant is given by $12 M \ell^{2}(4 m-M)$, and the function passes through the origin if $m=\frac{M}{3}$. Hence, noting that $0 \leq x \leq \ell$, the analysis can be split up into four cases: $m \in\left[0, \frac{M}{4}\right), m \in\left[\frac{M}{4}, \frac{M}{3}\right), m \in\left[\frac{M}{3}, M\right), m=M$, and $m \in(M, \infty)$. In the first 
three cases, the quadratic function is convex; the fourth case yields to a linear function; and in the fifth case the quadratic function is concave. In the first case, it is impossible to find any real $x$. The second case corresponds to $x \in\left[\frac{3 m-\sqrt{3 M(4 m-M)}}{3(M-m)} \ell, \frac{3 m+\sqrt{3 M(4 m-M)}}{3(M-m)} \ell\right]$. The third case corresponds to $x \in[0, \ell]$. The fourth case also corresponds to $x \in[0, \ell]$. In the fifth case, the quadratic function will never have any zeros, hence it also corresponds to $x \in$ $[0, \ell]$.

The second inequality is equivalent to $f(x)=3(M-m) x^{2}+6 m \ell x+(M-3 m) \ell^{2} \leq 0$. Its discriminant is given by $12 M \ell^{2}(4 m-M)$, and the function passes through the origin if $m=\frac{M}{3}$. Hence, noting that $0 \leq x \leq \ell$, the analysis can be split up into four cases: $m \in\left[0, \frac{M}{4}\right), m \in\left[\frac{M}{4}, \frac{M}{3}\right), m \in\left[\frac{M}{3}, M\right), m=M$, and $m \in(M, \infty)$. In the first three cases, the quadratic function is convex; the fourth case yields to a linear function; and in the fifth case the quadratic function is concave. In the first case, it is impossible to find any real $x$. In the second case, it is impossible to find any non-negative $x$. The third case corresponds to $x \in\left[0, \frac{-3 m+\sqrt{3(4 m-M)}}{3(M-m)} \ell\right]$. The fourth case corresponds to $x \in\left[0, \frac{t}{3}\right]$. In the fifth case, the quadratic function is concave, hence it corresponds to $x \in$ $\left[0, \frac{3 m-\sqrt{3 M(4 m-M)}}{3(m-M)} \ell\right]$.

Therefore, one local maximum can be found if either one of the following conditions is satisfied: $m \in\left[\frac{M}{4}, \frac{M}{3}\right)$ and $x \in\left[\frac{3 m-\sqrt{3 M(4 m-M)}}{3(M-m)} \ell, \frac{3 m+\sqrt{3 M(4 m-M)}}{3(M-m)} \ell\right]$, or $m \in\left[\frac{M}{3}, M\right)$ and $x \in\left(\frac{-3 m+\sqrt{3 M(4 m-M)}}{3(M-m)} \ell, \ell\right]$, or $m=M$ and $x \in\left(\frac{\ell}{3}, \ell\right]$, or $m \in(M, \infty)$ and $x \in\left(\frac{3 m-\sqrt{3 M(4 m-M)}}{3(m-M)} \ell, \ell\right]$; and two local maxima can be found if either one of the following conditions is satisfied: $m \in$ $\left[\frac{M}{3}, M\right)$ and $\left.x \in\left[0, \frac{-3 m+\sqrt{3 M(m-M)}}{3(M-m)}\right]\right]$, or $m=M$ and $x \in\left[0, \frac{\ell}{3}\right]$, or $m \in(M, \infty)$ and $x \in\left[0, \frac{3 m-\sqrt{3 M(4 m-M)}}{3(m-M)} \ell\right]$.

In the previous two generalisations, one may have thought that the reason why local maximum happens not at one of the rod's ends is due to the linear velocity the rod gains after the collision. But the result obtained in (24) tells us that even when the centre of mass of the rod is fixed $(x=0)$, the local maximum is attained not when the projectile hits one of the rod's ends, except when $m=\frac{M}{3}$.

What if $m \in\left[0, \frac{M}{4}\right)$, or $m \in\left[\frac{M}{4}, \frac{M}{3}\right)$ and $x \in\left[0, \frac{3 m-\sqrt{3 M(4 m-M)}}{3(M-m)} \ell\right)$ ? Then it would be impossible to find a local maximum for the angular speed, and global maxima should be taken, $a= \pm \ell$. The corresponding expressions for $\omega$ are $\omega_{\max }=\frac{2 m( \pm \ell-x)}{m( \pm \ell-x)^{2}+\left(\frac{1}{3} M \ell^{2}+M x^{2}\right)} v_{0}$. Intuitively, letting the projectile hit the rod at its end that is farther from the pivot, $a=-\ell$, should contribute to greater angular speed. To verify this,

$$
\begin{aligned}
& \frac{\ell-x}{m(\ell-x)^{2}+\left(\frac{1}{3} M \ell^{2}+M x^{2}\right)}-\frac{\ell+x}{m(\ell+x)^{2}+\left(\frac{1}{3} M \ell^{2}+M x^{2}\right)} \\
& =-\frac{2 x\left[(M+m) x^{2}+\left(\frac{1}{3} M-m\right) \ell^{2}\right]}{\left[m(\ell-x)^{2}+\left(\frac{1}{3} M \ell^{2}+M x^{2}\right)\right]\left[m(\ell+x)^{2}+\left(\frac{1}{3} M \ell^{2}+M x^{2}\right)\right]}
\end{aligned}
$$

which is obviously negative unless $x=0$. If $x=0$, the rod is symmetrical, and hence it does not matter which end of the rod the projectile hits.

From equation (22), it is obvious that the projectile will stop moving after the collision if $m(a-x)^{2}=\left(\frac{1}{3} M \ell^{2}+M x^{2}\right)$, or equivalently $a=x \pm \sqrt{\frac{M}{3 m}\left(\ell^{2}+3 x^{2}\right)}$. Comparing with equation (24), it tells us that local maxima occur when the projectile stops moving after the collision.

\section{Conclusion}

This paper generalises the case of a projectile hitting a stationary rod on a smooth table, by exploring the unevenness (non-uniformity) of the rigid body, the elasticity of the collision, as well as the case where the rod is pivoted. For the rod to attain the greatest angular speed after the collision, the projectile should hit the rod at a 
certain point, which is in general not the rod's end. This is true even if the rod is uniform, its centre of mass is pivoted, and the collision is elastic, as presented in the last section. All the results obtained have proven that intuition fails when it comes to specific physics problems that necessarily require mathematical approach.

In the first generalisation, the rod (mass $M$, length $2 \ell$, centre of mass at $x$ from its centre, moment of inertia about its centre of mass $I_{\mathrm{CM}}$ ) is assumed to be non-uniformly distributed, and the centre of mass of the rod is not at the centre of its dimension. Only elastic collision is being considered. To maximise the angular speed of the rod, the projectile (mass $\mathrm{m}$ ) should hit the rod perpendicularly at positions $a$ from the centre of the rod, where

$$
a= \begin{cases}x \pm \sqrt{\frac{(m+M) I_{\mathrm{CM}}}{M m}}, & \text { if } x \in\left[0, \ell-\sqrt{\left.\frac{(m+M) I_{\mathrm{CM}}}{M m}\right], \sqrt{\frac{(m+M) I_{\mathrm{CM}}}{M m}} \in[0, \ell)}\right. \\ x-\sqrt{\frac{(m+M) I_{\mathrm{CM}}}{M m}}, & \text { if } x \in\left[\sqrt{\left.\frac{(m+M) I_{\mathrm{CM}}}{M m}-\ell, \ell\right], \sqrt{\frac{(m+M) I_{\mathrm{CM}}}{M m}} \in[\ell, 2 \ell]}\right.\end{cases}
$$

The corresponding angular velocities are respectively given by

$$
\omega_{\max }= \begin{cases} \pm \sqrt{\frac{M m}{(m+M) I_{\mathrm{CM}}}} v_{0}, & \text { if } x \in\left[0, \ell-\sqrt{\left.\frac{(m+M) I_{\mathrm{CM}}}{M m}\right], \sqrt{\frac{(m+M) I_{\mathrm{CM}}}{M m}} \in[0, \ell)}\right. \\ -\sqrt{\frac{M m}{(m+M) I_{\mathrm{CM}}}} v_{0}, & \text { if } x \in\left[\sqrt{\frac{(m+M) I_{\mathrm{CM}}}{M m}}-\ell, \ell\right], \sqrt{\frac{(m+M) I_{\mathrm{CM}}}{M m}} \in[\ell, 2 \ell]\end{cases}
$$

Figure 6 illustrates the behaviour of $\frac{\omega \ell}{2 \mathrm{v}_{0}}$ as a function of $\frac{a}{\ell}$ in this scenario, for $\sqrt{\frac{(\mathrm{m}+\mathrm{M}) \mathrm{I}_{\mathrm{CM}}}{\mathrm{Mm}}}=0.8, x=0.1 \ell$; and for $\sqrt{\frac{(m+M) I_{C M}}{M m}}=1.3, x=0.7 \ell$.

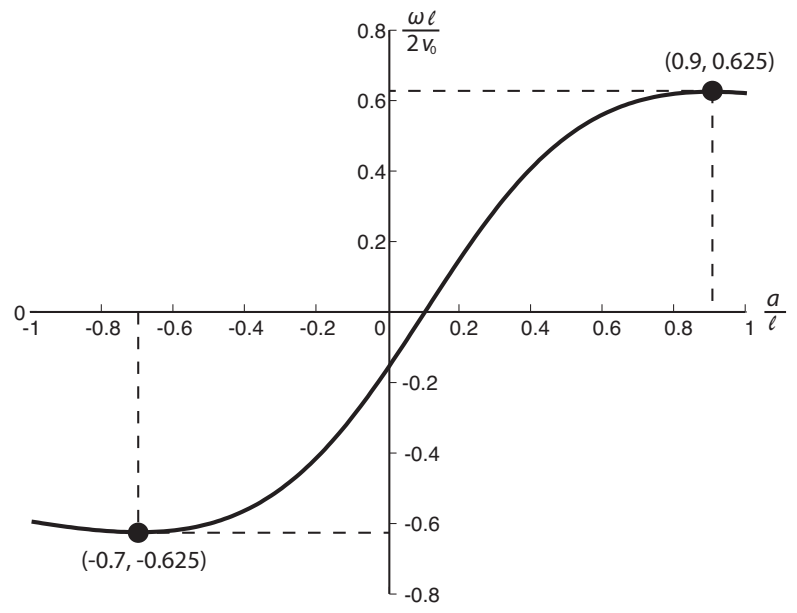

(a)

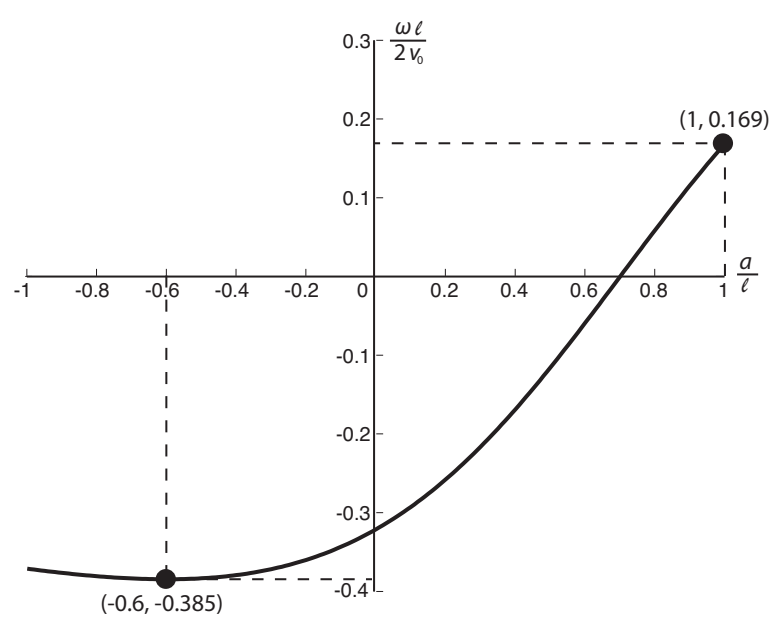

(b)

Figure 6. Graph of $\frac{\omega \ell}{2 v_{0}}$ vs $\frac{a}{\ell}$ for: (a) $\sqrt{\frac{(m+M) I_{\mathrm{CM}}}{M m}}=0.8 \ell, x=0.1 \ell ;$ (b) $\sqrt{\frac{(m+M) I_{\mathrm{CM}}}{M m}}=1.3 \ell, x=0.7 \ell$

In the second generalisation, the rod is assumed to be uniformly distributed (mass $M$, length $2 \ell$, moment of inertia about its centre of mass $\left.\frac{1}{3} M \ell^{2}\right)$. The collision is assumed to be dissipative where $\varepsilon=\frac{(V-v)+3 v \frac{a^{2}}{\ell^{2}}}{v_{0}} \in[0,1]$. To maximise the angular speed, the projectile (mass $\mathrm{m}$ ) should hit the rod perpendicularly at positions $a$ from the centre of the rod, where

$$
a=\left\{\begin{array}{rr} 
\pm \ell \sqrt{\frac{M+m}{3 m},} & M \leq 2 m \\
\pm \ell, & M>2 m
\end{array}\right.
$$

The corresponding angular velocities are respectively given by 


$$
\omega_{\max }= \begin{cases} \pm \sqrt{\frac{3 m}{M+m} \frac{(1+\varepsilon) v_{0}}{2 \ell},} & M \leq 2 m \\ \pm \frac{3 m}{4 m+M} \frac{(1+\varepsilon) v_{0}}{\ell}, & M>2 m\end{cases}
$$

Figure 7 illustrates the behaviour of $\frac{\omega \ell}{3(1+\varepsilon) v_{0}}$ as a function of $\frac{a}{\ell}$ in this scenario, for $\frac{M}{m}=0.47$ and $\frac{M}{m}=5.75$.

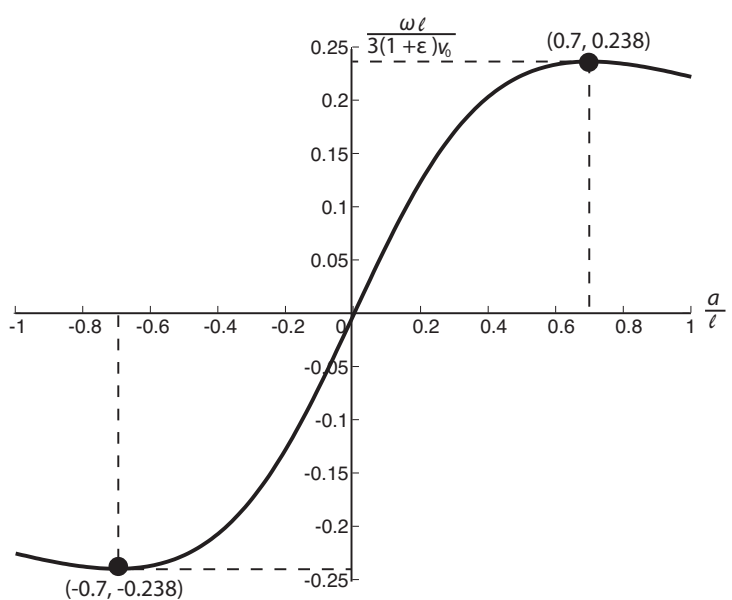

(a)

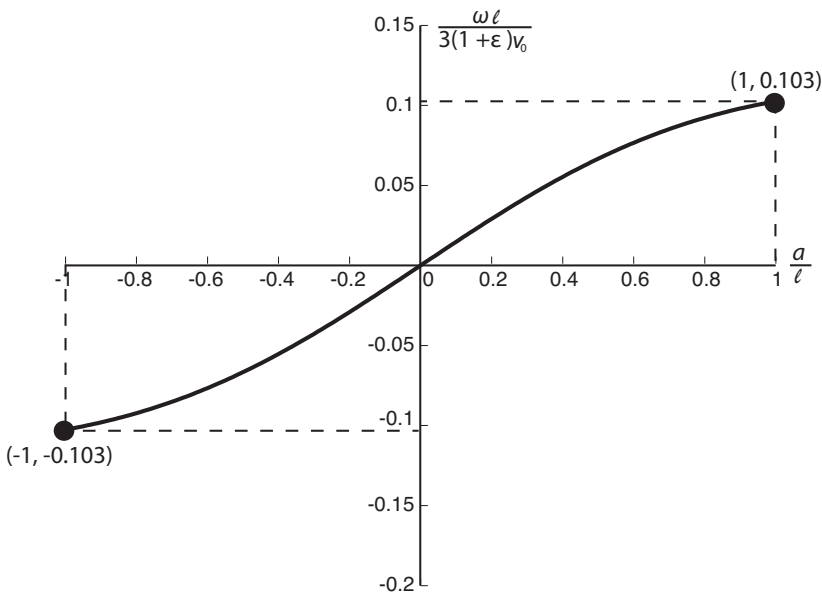

(b)

Figure 7. Graph of $\frac{\omega \ell}{3(1+\varepsilon) v_{0}}$ vs $\frac{a}{\ell}$ for: (a) $\frac{M}{m}=0.47$; (b) $\frac{M}{m}=5.75$

In the third generalisation, the rod is assumed to be uniformly distributed (mass $M$, length $2 \ell$, moment of inertia about its centre of mass $\left.\frac{1}{3} M \ell^{2}\right)$. The collision is assumed to be elastic. A pivot is being introduced in the system at distance $x$ from the centre of the rod. To maximise the angular speed of the rod, the projectile (mass $m$ ) should hit it perpendicularly at positions $a$ from the centre of the rod, where $a=x \pm \sqrt{\frac{M}{3 m}\left(\ell^{2}+3 x^{2}\right)}$ and $\omega_{\max }= \pm \sqrt{\frac{m}{\frac{1}{3} M \ell^{2}+M x^{2}}} v_{0}$ if any of the followings is satisfied:

$$
\left\{\begin{array}{c}
m \in\left[\frac{M}{3}, M\right) \text { and } x \in\left[0, \frac{-3 m+\sqrt{3 M(4 m-M)}}{3(M-m)} \ell\right] \\
m=M \text { and } x \in\left[0, \frac{\ell}{3}\right] \\
m \in(M, \infty) \text { and } x \in\left[0, \frac{3 m-\sqrt{3 M(4 m-M)}}{3(m-M)} \ell\right]
\end{array}\right.
$$

$a=x-\sqrt{\frac{M}{3 m}\left(\ell^{2}+3 x^{2}\right)}$ and $\omega_{\max }=-\sqrt{\frac{m}{\frac{1}{3} M \ell^{2}+M x^{2}}} v_{0}$ if any of the followings is satisfied:

$$
\left\{\begin{array}{c}
m \in\left[\frac{M}{4}, \frac{M}{3}\right) \text { and } x \in\left[\frac{3 m-\sqrt{3 M(4 m-M)}}{3(M-m)} \ell, \frac{3 m+\sqrt{3 M(4 m-M)}}{3(M-m)} \ell\right] \\
m \in\left[\frac{M}{3}, M\right) \text { and } x \in\left(\frac{-3 m+\sqrt{3 M(4 m-M)}}{3(M-m)} \ell, \ell\right] \\
m=M \text { and } x \in\left(\frac{\ell}{3}, \ell\right] \\
m \in(M, \infty) \text { and } x \in\left(\frac{3 m-\sqrt{3 M(4 m-M)}}{3(m-M)} \ell, \ell\right]
\end{array}\right.
$$


$a=-\ell$ and $\omega_{\max }=-\frac{2 m(\ell+x)}{m(\ell+x)^{2}+\left(\frac{1}{3} M \ell^{2}+M x^{2}\right)} v_{0}$ if any of the followings is satisfied:

$$
\left\{\begin{array}{c}
m \in\left[0, \frac{M}{4}\right) \\
m \in\left[\frac{M}{4}, \frac{M}{3}\right) \text { and } x \in\left[0, \frac{3 m-\sqrt{3 M(4 m-M)}}{3(M-m)} \ell\right)
\end{array}\right.
$$

Figure 8 illustrates the behaviour of $\frac{\omega \ell}{2 v_{0}}$ as a function of $\frac{a}{\ell}$ in this scenario, for $m=M, x=0.2 \ell ; m=0.5 M$, $x=0.5 \ell ;$ and $m=0.1 M, x=0.5 \ell$.

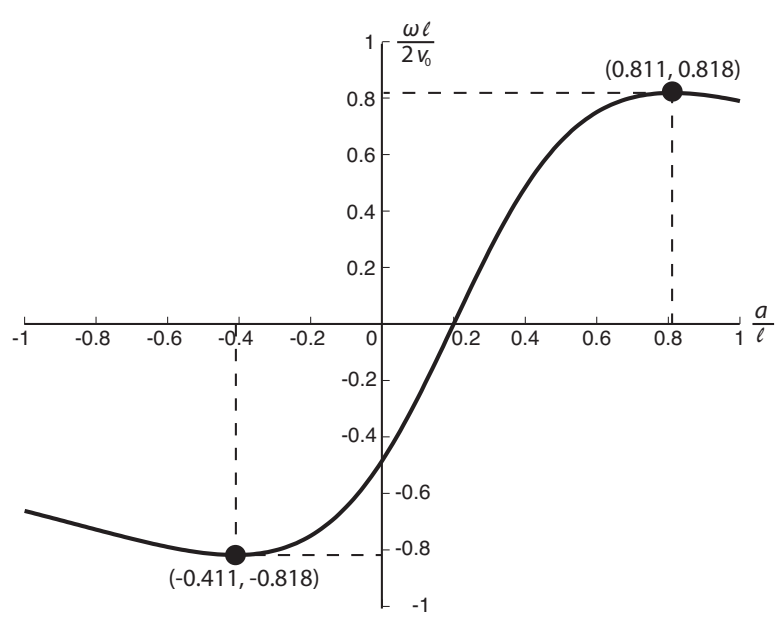

(a)

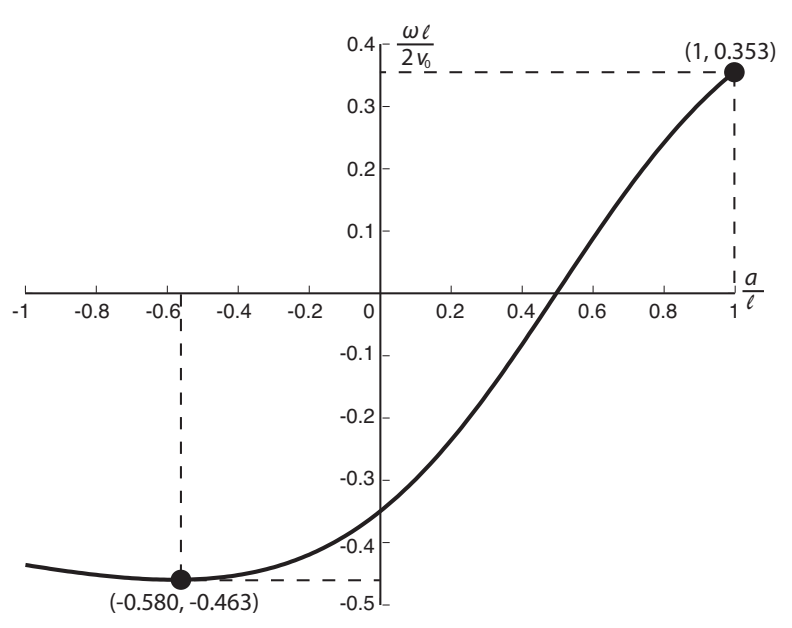

(b)

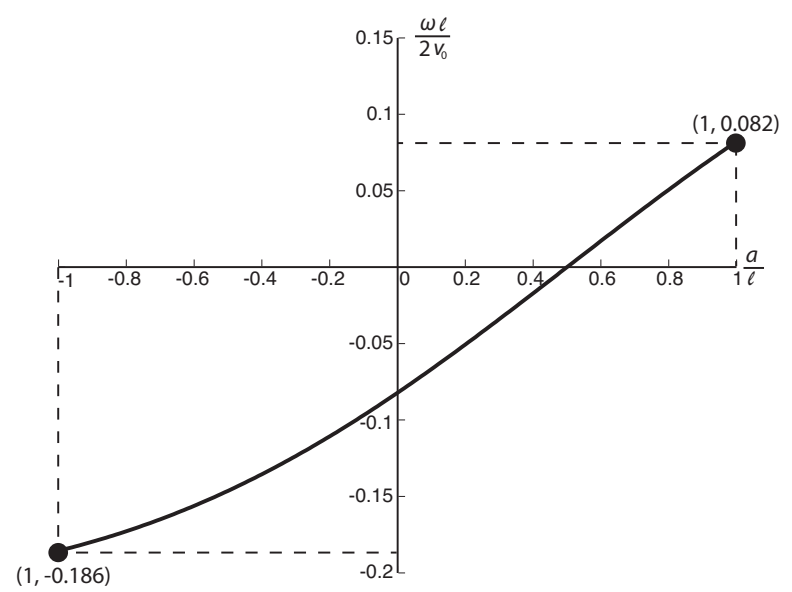

(c)

Figure 8. Graph of $\frac{\omega \ell}{2 \mathrm{v}_{0}}$ vs $\frac{a}{\ell}$ for: (a) $m=M, x=0.2 \ell$; (b) $m=0.5 M, x=0.5 \ell$; (c) $m=0.1 M, x=0.5 \ell$

In general, the local maxima occur not at the rod's end, regardless of the generalisation of the case. This is counterintuitive as it goes against our daily experience with doors and levers. The underlying concepts behind the problem presented are simple, and students with good conceptual understanding may come out with inaccurate analysis as they approach it intuitively. For relatively advanced physics problems, mathematical formulas therefore should not be avoided. 


\section{Acknowledgement}

The author, Bernard Ricardo, would like to thank NUS High School of Mathematics and Science, Singapore, for supporting this work, and A/P Paul Lee from the National Institute of Education, Singapore, for supervising this work.

\section{References}

Feynman, R. P., Leighton, R. B., \& Sands, M. (2013). The Feynman Lectures on Physics (Desktop Edition Volume I, Vol. 1). Basic Books.

Lemos, N. A. (2008). Failure of intuition in elementary rigid body dynamics. European Journal of Physics, 29(2), N1. http://dx.doi.org/10.1088/0143-0807/29/2/n01

Podolsky, B. (1966). Conservation of Angular Momentum. American Journal of Physics, 34(1), 42-45. http://dx.doi.org/10.1119/1.1972743

Resnick, D. H., \& Kenneth, S. K. (2001). Physics Volume I (5th ed., pp. 356-357). New York: Wiley.

Ricardo, B. (2015). Using scaling to compute moments of inertia of symmetric objects. European Journal of Physics, 36(5), 055003. http://dx.doi.org/10.1088/0143-0807/36/5/055003

Ricardo, B., \& Lee, P. (2015). Maximizing kinetic energy transfer in one-dimensional many-body collisions. European Journal of Physics, 36(2), 025013. http://dx.doi.org/10.1088/0143-0807/36/2/025013

Thornton, C. (1997). Coefficient of restitution for collinear collisions of elastic-perfectly plastic spheres. Journal of Applied Mechanics, 64(2), 383-386. http://dx.doi.org/10.1115/1.2787319

\section{Copyrights}

Copyright for this article is retained by the author(s), with first publication rights granted to the journal.

This is an open-access article distributed under the terms and conditions of the Creative Commons Attribution license (http://creativecommons.org/licenses/by/3.0/). 